\title{
Tumor suppressor let-7a inhibits breast cancer cell proliferation, migration and invasion by targeting MAGE-A1
}

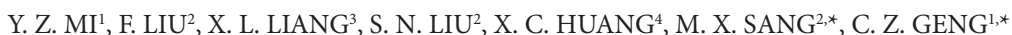

${ }^{1}$ Breast Center Department, The Fourth Hospital of Hebei Medical University, Hebei Medical University, Shijiazhuang, China; ${ }^{2}$ Research Center Department, The Fourth Hospital of Hebei Medical University, Hebei Medical University, Shijiazhuang, China; ${ }^{3}$ Laboratory of Pathology, Hebei Cancer Institute, The Fourth Hospital of Hebei Medical University, Shijiazhuang, China; ${ }^{4}$ Department of General Surgery, The Fourth Hospital of Hebei Medical University, Shijiazhuang, China

*Correspondence: mxsang@hotmail.com, cuizhigeng@hotmail.com

Received March 2, 2018 / Accepted May 21, 2018

\begin{abstract}
Let-7 was one of the earliest discovered miRNAs and while it reportedly acts as a tumor suppressor in various solid tumors, its function in breast cancer has not been fully studied. Therefore, we examined let-7a and MAGE-A1 expression in breast tissues by qRT-PCR and found that let-7a expression significantly correlates with larger tumor size, higher histological grade $(\mathrm{p}<0.05)$ and is significantly lower in patients with Her-2-positive cancers and Ki-67 $>14 \%(\mathrm{p}=0.028$ and $\mathrm{p}=0.023)$. MAGE-A1 expression incidence is $50.8 \%(33 / 65)$ and it inversely correlates with let-7a expression $(\mathrm{p}=0.008)$. let-7a inhibition of breast cancer cell proliferation, migration and invasion was also observed in in vitro cell culture experiments, and dual-luciferase reporter assays showed that melanoma-associated antigen A1 (MAGE-A1) was its target gene; the target comprised bases 451-457 of the 3'UTR region of the MAGE-A1 mRNA. RT-qPCR and Western blot analyses showed that let-7a inhibited MAGE-A1 expression at both the nucleic acid and protein levels. In our final co-transfection experiment, we targeted MAGE-A1 in a breast cancer cell line and observed that let-7a inhibited cell proliferation, migration and invasion. These combined results confirm that let-7a functions as a tumor suppressor by targeting MAGE-A1 in breast cancer and it therefore provides a novel target in breast cancer clinical treatment.
\end{abstract}

Key words: breast cancer, miRNA, let-7a, MAGE-A1

Breast cancer is the most common cancer, and its metastatic progression is a complex process [1-2]. While surgery and chemotherapy are the current primary clinical treatments, many patients who undergo chemotherapy have early tumor recurrence, metastases and poor prognoses. Since precise treatment is now sought for breast cancer, exploring targeted treatments has become especially vital.

MiRNAs are novel molecules involved in gene regulation and are small non-coding RNAs molecules approximately 22 nucleotides in length which discretely regulate target gene expression by post-transcriptional action [3]. Degradation or translational inhibition of the target mRNA can be effected by sequence-specific base pairing of a miRNA with the 3' untranslated region (3'UTR) of the target mRNA in the RNA-induced silencing complex [4-5]. miRNA involvement in gene regulation is the most fundamental step in genetic programs which affect cell proliferation, differentiation and apoptosis.

The let-7a MiRNA has received substantial attention because of its aberrant expression in human cancers. Multiple studies have reported that let-7a is expressed poorly in breast cancer [6], lung cancer [7], gastric tumors [8], colorectal cancer [9] and laryngeal squamous cancer [10]. This poor let-7a expression may also lead to poor prognosis for lung cancer patients [7]. Recent studies have also indicated that let-7a is a tumor suppressor and represses the expression of high-mobility group A (HMGA), RAS and c-MYC which are critical oncogenes in tumorigenesis, proliferation and invasion $[6,11-13]$. However, whether let-7a regulates other oncogenes as a tumor suppressor in breast cancer requires further study.

The melanoma-associated antigen (MAGE) family is characterized by a unique pattern of tissue expression, and its members are aberrantly expressed in a wide variety of tumors; MAGEs are not expressed in normal adult tissues, except for immuno-privileged germ-line tissues [14]. Since its first discovery in 1991 [15], dozens of MAGE genes have been identified and these are divided into two subtypes: MAGE-I and MAGE-II.

MAGE-I comprises three subfamilies, namely, MAGE-A, MAGE-B and MAGE-C. Of these, the MAGE-A subfamily 
is located at the q28 site of the human $\mathrm{X}$ chromosome and includes 12 members; MAGE-A1 MAGE-A12 [16]. Studies have shown that the A1, A2, A3, A4, A6, and A12 MAGE-A sub-family members are expressed in breast cancer to varying degrees (6 69\%), with higher expression in patients with higher recurrence risk [17]. Retrospective studies have shown that the mortality rate of MAGE-A1-positive breast cancer patients is significantly higher than that of MAGE-A1-negative patients [18] and further analysis revealed that the serum levels of MAGE-A1 serum levels and let-7b were significantly higher in patients with invasive carcinomas than in healthy women and those with benign breast disease [19]. Thus, MAGE-A1 expression increases the risk of recurrence and mortality in breast cancer patients and is related to the expression of let- 7 family members. Based on the above findings, this study explores interactions between let-7a and MAGE-A1.

Here, we found that let-7 expression was associated with poor clinical-pathological characteristics in breast cancer patients and that it negatively correlates with MAGE-A1 expression in breast cancer tissues. Functionally, let-7a inhibits breast cancer cell proliferation, migration and invasion by targeting MAGE-A1.

\section{Patients and methods}

Breast cancer tissues. Tumor tissues $(n=65)$ with complete clinical and histopathological data were obtained from the department of Breast Center, the fourth hospital of Hebei Medical University from 2016 to 2017. The patients in the study provided written informed consent for tissue sample analysis and did not receive pre-operative treatment (including chemotherapy, radiotherapy, targeted therapy and endocrine therapy). The protocol of the study was approved by the Institutional Ethics Committee at Fourth Hospital of Hebei Medical University.

Cell lines and transfection. The human breast cancer cell lines MDA-MB-231 (human non-hormone-dependent breast cancer cell line) and MCF-7 (human hormone-dependent breast cancer cell line) were obtained from American Type Culture Collection (ATCC, USA). Both cell lines were cultured in DMEM (Gibco, USA) containing 10\% fetal bovine serum and $100 \mathrm{IU} / \mathrm{ml}$ penicillin/streptomycin at $37^{\circ} \mathrm{C}$ in an incubator containing $5 \% \mathrm{CO}_{2}$ and humidified air. The let- $7 \mathrm{a}$ mimics, let-7a inhibitor, and negative control were synthesized and purified by GenePharma (Shanghai, China). The miRNA mimics and inhibitor complexes were transfected at working concentrations of $100 \mathrm{nM}$ by HiPerFect Transfection Reagent (QIAGEN, GRE) and co-transfected with miRNA mimics and MAGE-A1 expression vector by FuGENE $^{\bullet} \mathrm{HD}$ (Promega, USA).

RNA isolation and qRT-PCR. Total RNA was isolated from collected cells by TRIzol reagent (Invitrogen) according to the manufacturer's instructions. For qPCR analysis of let-7a, $2 \mu \mathrm{g}$ RNA was reverse transcribed into cDNA using the miDETECT A Track $^{\mathrm{mm}}$ miRNA qRT-PCR (Ribobio,
China), and the reactions were performed by GoTaq ${ }^{\circ} \mathrm{qPCR}$ Master Mix on the Quant studio DX real-time PCR System. The amount of let-7a was obtained by normalizing the let-7a level to small nuclear U6 snRNA as the control. For qPCR analysis of MAGE-A1, $2 \mu \mathrm{g}$ RNA was reverse transcribed into cDNA using the Reverse Transcription System kit (Promega, USA), and the reactions were carried out in triplicate using the GoTaq ${ }^{\circledR}$ PCR Master Mix on the Quant studio DX realtime PCR System. The amount of MAGE-A1 was determined with GAPDH as the control. The specific primers for human MAGE-A1 were 5'-GTGGTCCTAAGATCTACCAAGCA-3' (forward) and 5'-AGGGCAGCAGGTAGGAGTG-3' (reverse), and the specific primers for human GAPDH were 5'-CGCTGAGTACGTCGTGGAGTC-3' (forward) and 5'-GCTGATGATCTTGAGGCTGTTGTC-3' (reverse). The annealing temperature for both genes was $58^{\circ} \mathrm{C}$. All experiments were performed independently in triplicate and threshold cycle $(\mathrm{Ct})$ values were analyzed by comparative $\mathrm{Ct}$ $(\Delta \Delta \mathrm{Ct})$.

Western blot. Total protein from the cell lines was extracted in RIPA lysis buffer, and equal $30 \mu \mathrm{g}$ amounts of protein lysates were separated by $12 \%$ SDS-polyacrylamide gel electrophoresis and transferred to polyvinylidene fluoride (PVDF) membranes (Beyotime). The membranes were blocked with 5\% non-fat milk for $1 \mathrm{~h}$. After blocking, the membrane was incubated with specific testing antibodies for MAGE-A1 (1:500; Santa Cruz, USA) and $\beta$-actin (1:1000; Proteintech, USA). After overnight blotting at $4{ }^{\circ} \mathrm{C}$, the membranes were washed with TBST and then incubated with HRP-linked secondary antibodies (1:10 000; ZSGB-BIO, China). The protein bands were developed with chemiluminescence (ECL) reagents (Beyotime).

3'-UTR luciferase plasmid construct and analysis. The 3'-UTR of MAGE-A1 was cloned, and the putative sites that could bind to let- $7 \mathrm{a}$ were mutated. The mutant and wild-type sequences were separately cloned into the pmirGLO vector (Promega, USA). Cos-7 cells were co-transfected with the constructed vectors and let-7a mimic. After $48 \mathrm{~h}$ of transfection, the firefly and Renilla dual-luciferase testing system (Promega, USA) was used to measure and calculate luciferase activity.

Cell proliferation assay. MDA-MB-231 and MCF-7 cells were transfected with let-7a mimic and negative control or let-7a inhibitor and inhibitor negative control and were seeded into 96 -well plates at $24 \mathrm{~h}$ after transfection $\left(3 \times 10^{3}\right.$ cells/well). After an additional $24 \mathrm{~h}, 48 \mathrm{~h}$, or $72 \mathrm{~h}$, cells were

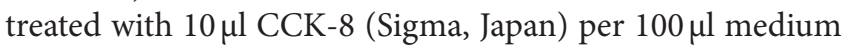
and cell proliferation was then assessed by recording the absorbance at $450 \mathrm{~nm}$ (OD value).

Cell invasion assay. MDA-MB-231 and MCF-7 cells were subjected to the above treatments. After $24 \mathrm{~h}$ transfection, $5 \times 10^{5}$ cells were seeded into chambers $(8 \mu \mathrm{m}$, Corning) containing $20 \mu \mathrm{l}$ diluted matrigel (matrigel:DMEM $=7: 1$ ). A total of $0.2 \mathrm{ml}$ serum-free DMEM was added to the upper chamber and DMEM containing 10\% fetal bovine serum 
was added to the lower one for $24 \mathrm{~h}$ (MDA-MB-231) or $48 \mathrm{~h}$ (MCF-7). The invasive cells were fixed and stained with $0.1 \%$ crystal violet. Three low-magnification areas $(\times 200)$ were randomly selected and cell numbers were determined. The experiment was performed in triplicate.

Cell migration assay. For horizontal migration, the tumor cells were seeded in 6-well plates and transfected with let-7a mimic, let-7a inhibitor and negative control, or co-transfected with miRNA mimic and the MAGE-Al expression vector until the cells reached $100 \%$ confluence. The cell layer was then scratched through the central axis using a sterile plastic tip and loose cells were washed away with PBS. Wound healing was observed and photographed at $0 \mathrm{~h}$ and $24 \mathrm{~h}$ in the same selected microscopic fields $(\times 100)$, and transwells without matrigel were used for vertical migration. A total of $2 \times 10^{5}$ cells were seeded in the chambers; and $0.2 \mathrm{ml}$ serumfree DMEM was added to the upper chamber, and DMEM containing $10 \%$ fetal bovine serum was added to the lower chamber for $20 \mathrm{~h}$ (MDA-MB-231) or $24 \mathrm{~h}$ (MCF-7).

Statistical analysis. Data is expressed as mean \pm standard deviation (SD), and statistical analysis was performed with SPSS 21. The relationship between two groups was analyzed by chi-square and the relationship between two or more variables was tested by one-way ANOVA.

\section{Results}

Expression and relationship of let-7a and MAGE-A1 in breast cancer tissues. We used real-time PCR to determine the let-7a and MAGE-A1 levels in 65 breast cancer tissues, all of which expressed let-7a. For data analysis, we categorized all breast cancer patients into let-7a high and low expression groups using the quartet of let-7a expression as the cutoff threshold in all breast cancer patients. As shown in Table 1, the let-7a expression level was significantly lower in patients with tumors $>2 \mathrm{~cm}$ than in patients with tumors $\leq 2 \mathrm{~cm}$ $(\mathrm{p}=0.049)$ and significantly lower in patients with histolog-

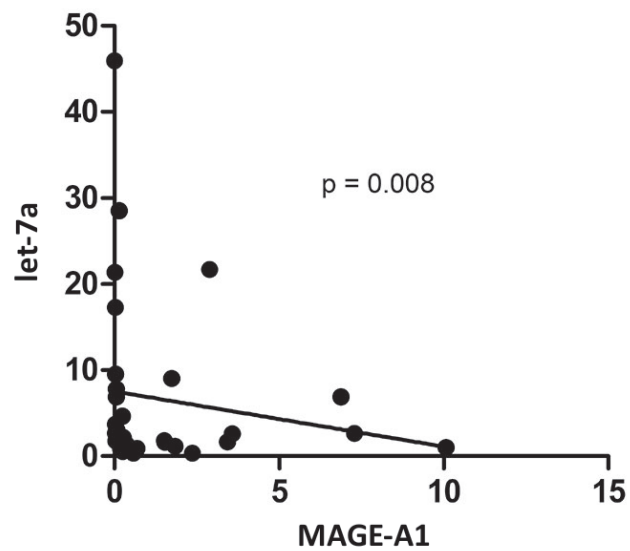

Figure 1. The relationship between let-7a and MAGE-A1 in the whole cohort of breast cancer patients.
Table 1. Let-7a and MAGE-A1 expression and clinical-pathological parameters in breast cancer.

\begin{tabular}{|c|c|c|c|c|c|c|c|}
\hline \multirow{2}{*}{ Clinical features } & \multirow{2}{*}{$\mathbf{N}$} & \multicolumn{3}{|c|}{ Let-7a } & \multicolumn{3}{|c|}{ MAGE-A1 } \\
\hline & & High & Low & p-value & + & - & p-value \\
\hline Age (years) & & & & 0.543 & & & 0.265 \\
\hline$<50$ & 32 & 15 & 17 & & 14 & 18 & \\
\hline$\geq 50$ & 33 & 13 & 20 & & 19 & 14 & \\
\hline BMI & & & & 0.225 & & & 0.543 \\
\hline$<25$ & 24 & 8 & 16 & & 11 & 13 & \\
\hline$\geq 25$ & 41 & 20 & 21 & & 22 & 19 & \\
\hline Menopausal state & & & & 0.914 & & & 0.903 \\
\hline Premenopause & 33 & 14 & 19 & & 17 & 16 & \\
\hline Postmenopause & 32 & 14 & 18 & & 16 & 16 & \\
\hline Tumor size (cm) & & & & 0.049 & & & 0.168 \\
\hline$\leq 2$ & 35 & 19 & 16 & & 15 & 20 & \\
\hline$>2$ & 30 & 9 & 21 & & 18 & 12 & \\
\hline Axillary lymph node & & & & 0.409 & & & 0.531 \\
\hline- & 34 & 13 & 21 & & 16 & 18 & \\
\hline+ & 31 & 15 & 16 & & 17 & 14 & \\
\hline TNM stage & & & & 0.150 & & & 0.914 \\
\hline I & 17 & 10 & 7 & & 9 & 8 & \\
\hline II & 39 & 13 & 26 & & 20 & 19 & \\
\hline III & 9 & 5 & 4 & & 4 & 5 & \\
\hline Histological grade & & & & 0.041 & & & 0.384 \\
\hline G1-2 & 42 & 22 & 20 & & 23 & 19 & \\
\hline G3 & 23 & 6 & 17 & & 10 & 13 & \\
\hline Subtype & & & & 0.095 & & & 0.683 \\
\hline Luminal A or B & 40 & 22 & 18 & & 22 & 18 & \\
\hline HER2 positive & 14 & 3 & 11 & & 6 & 8 & \\
\hline Triple negative & 11 & 5 & 6 & & 5 & 6 & \\
\hline ER & & & & 0.154 & & & 0.388 \\
\hline - & 25 & 8 & 17 & & 11 & 14 & \\
\hline+ & 40 & 20 & 20 & & 22 & 18 & \\
\hline $\mathrm{PR}$ & & & & 0.102 & & & 0.543 \\
\hline- & 26 & 8 & 18 & & 12 & 14 & \\
\hline+ & 39 & 20 & 19 & & 21 & 18 & \\
\hline HER-2 & & & & 0.028 & & & 0.357 \\
\hline- & 48 & 26 & 22 & & 26 & 22 & \\
\hline+ & 17 & 4 & 13 & & 7 & 10 & \\
\hline $\mathrm{Ki}-67$ & & & & 0.023 & & & 0.943 \\
\hline$\leq 14 \%$ & 16 & 11 & 5 & & 8 & 8 & \\
\hline$>14 \%$ & 49 & 17 & 30 & & 25 & 24 & \\
\hline
\end{tabular}

ical grade III tumors than in patients with histological grade II or I ( $\mathrm{p}=0.041)$. The let-7a expression level was also significantly lower in patients with Her-2-positive tumors and Ki- $67>14 \%$ ( $\mathrm{p}=0.028$ and $\mathrm{p}=0.023$, respectively). There were no significant differences in age, BMI, menopausal state, clinical stage, lymph node metastasis, immunohistochemical typing, ER expression or PR expression. Thirty-three breast cancer tissues expressed MAGE-A1, and the rate was $50.8 \%$ (33/65). In MAGE-A1-positive samples, Spearman's rank-order correlation analysis was used and this showed that MAGE-A1 expression negatively correlates with let-7a expression $(\mathrm{p}=0.008$, Figure 1$)$. 
Let-7a inhibits breast cancer cell proliferation, migration and invasion. We selected MDA-MB-231 and MCF-7 cell lines for breast cancer research and measured let-7a relative expression (Figure 2A). We then transfected cells with mimics (MDA-MB-231) or inhibitor (MCF-7 dependent on let-7a expression levels, and CCK-8 assays revealed significant inhibition of cell proliferation in the let-7a mimic-transfected cells and large cell proliferation increase in the let-7a inhibitor-transfected cells compared to the let-7a mimic NC and inhibitor NC-transfected cells (Figures 3A, 3B). The cell scratch assays showed that the cells transfected with let-7a mimics migrated slowly and that those transfected with let-7a inhibitor migrated rapidly compared to the corresponding NC-transfected cells (Figures 3C, 3D). Transwell assays (without matrigel) showed that the number of tumor cells after treatment with let-7a mimics was significantly less than that after treatment with the mimic NC, and that the number of tumor cells after treatment with let-7a inhibitor was significantly higher than that after treatment with the inhibitor NC (Figures 3E, $3 \mathrm{~F})$, This demonstrates that let-7a inhibited breast cancer cell migration.

Matrigel invasion assays indicated that the invading cell numbers were significantly decreased in let-7a mimictransfected cells compared to control cells and significantly increased in the let-7a inhibitor-transfected cells compared to controls (Figures 3G, 3H). This established that let-7a inhibits tumor cell invasion.

MAGE-A1 is a direct target gene of let-7a. We transfected let-7a mimics and inhibitor into breast cancer cell lines (MDA-MB-231 and MCF-7). The MAGE-A1 expression level was verified by real-time PCR and Western blot. The results confirmed that let-7a expression negatively correlates with MAGE-A1 expression at the nucleic acid and protein levels (Figures $2 \mathrm{~B}, 2 \mathrm{C}, 2 \mathrm{D}$ ). Then, we predicated the putative target sites of let-7a in the 3'-UTR of MAGE-A1 using TargetScan. To determine whether MAGE-A1 was a direct target of let-7a, we performed luciferase reporter assays with a vector encoding the total sequence of the MAGE-A1 mRNA 3'-UTR or with a vector encoding its mutant 3'-UTR. The putative target site of let-7a in the 3'-UTR of MAGE-A1 is illustrated in Figure 2E, and results showed that luciferase activity was significantly reduced by the co-transfection of the entire 3'-UTR of MAGE-A1 and let-7a mimic, whereas the mutation of the let-7a target site blocked decreased luciferase activity (Figure 2F). These results clearly indicate that MAGE-A1 is a direct target of let-7a.

Let-7a inhibits breast cancer cell proliferation, migration and invasion by targeting MAGE-A1. The inhibitory effects of let-7a on cell proliferation, migration and invasion were rescued by co-expression of the MAGE-A1 vector in MDA-MB-231 cells (Figure 4). These findings indicate that let-7a inhibits cell proliferation, migration and invasion by regulating MAGE-A1 expression in MDA-MB-231 cells.

\section{Discussion}

The let-7 gene was originally discovered by Reinhart et al. in 2000 in a study on nematodes. The final product is 22 bases, and the let-7 gene is highly conserved and widely present in many animals [20]. The Let-7 family has 13 members, and these are located on nine different chromosomes [21]. Like most miRNAs, the post-transcriptional regulatory functions of let-7 are performed via its specific binding to the 3'UTR base sequence of the mRNA of a target gene. When the bases of the two are highly complementary, target gene mRNA can be degraded, and if these bases are not highly complementary, the target can be impeded during the process of gene mRNA translation [4-5].

The expression of let-7a, a member of the let-7 family, is decreased in breast, colon and thyroid cancer tissues compared to their normal tissues. When let-7a is overexpressed in the corresponding tumor cell line, the ability of the cell to proliferate, migrate, and invade is reduced $[6,9]$. Let-7a is associated with pathological tumor stages, including the TNM stage and lymph node metastasis in papillary thyroid carcinoma patients [22]. Erturk et al. found that let-7a expression was 4.67-fold reduced in triple negative breast cancer (TNBC) tissues compared to normal tissues [23]. However, that study did not investigate the differences in let-7a expression in different breast cancer subtypes.

Many studies addressing the targets of let-7a have been conducted. The main targets are RAS, HMGA1/HMGA2, c-myc and Lin28 [6, 11-13, 24], and other target genes remain to be studied. Let-7a can negatively regulate key oncogenes and serve as a suppressor in a variety of malignant tumors. The tumor suppressive role of let-7a is widely recognized, and the high expression of let-7a may serve as a new breakthrough for cancer gene therapy. Trang [25] used the lentivirus method to exogenously import let-7 into a mouse model of non-small cell lung cancer and found that $66 \%$ of the tumors were reduced to varying degrees. This showed successful application of let-7 in the treatment of non-small cell lung cancer and made a crucial step in clinical application.

MAGE-A family members are highly expressed in cancers and play critical roles in tumorigenesis [26]. MAGE-A1, a member of the let-7 family, is abnormally expressed in several types of tumors, including breast [17-18, 27], colorectal [28] and bladder cancers [29] and also glioma [30]. Our group reported that MAGE-A's are not expressed in breast cancer adjacent tissues and that the positivity rate in breast cancer tissues was $49.17 \%$. Their expression is associated with a high histological grade and axillary lymph node metastasis, and MAGE-A expression is significantly higher in TNBC and may be responsible for its high aggressiveness and epithelial mesenchymal transition (EMT) [27].

Our study first examined the expression of let-7a and MAGE-A1 mRNA in 65 breast cancers tissues, and found that the let-7a expression levels were lower in patients with tumors $>2 \mathrm{~cm}$ than those $\leq 2 \mathrm{~cm}$ and lower in patients with 
A

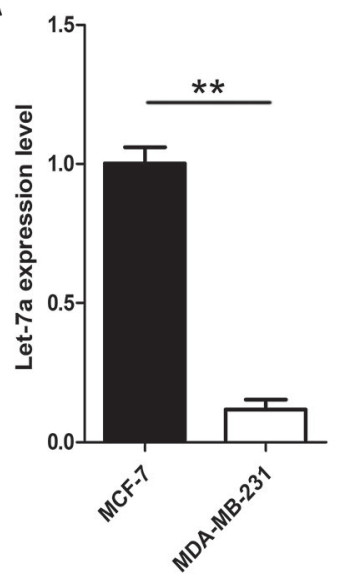

B

MDA-MB-231

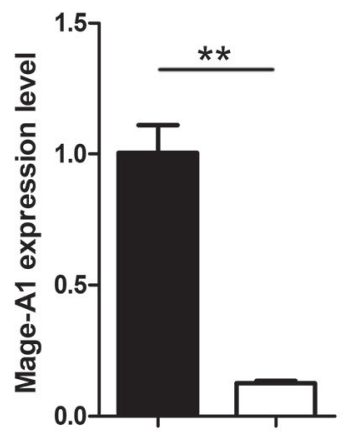

C
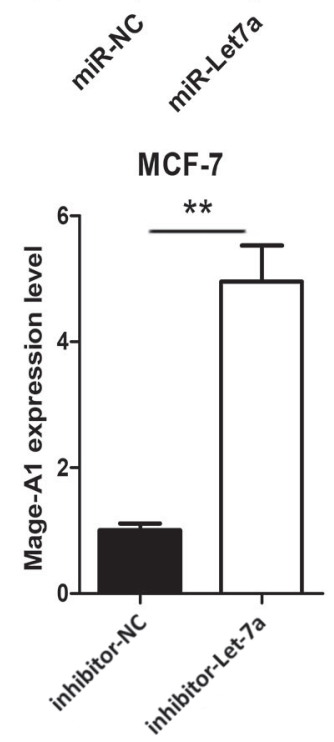

D
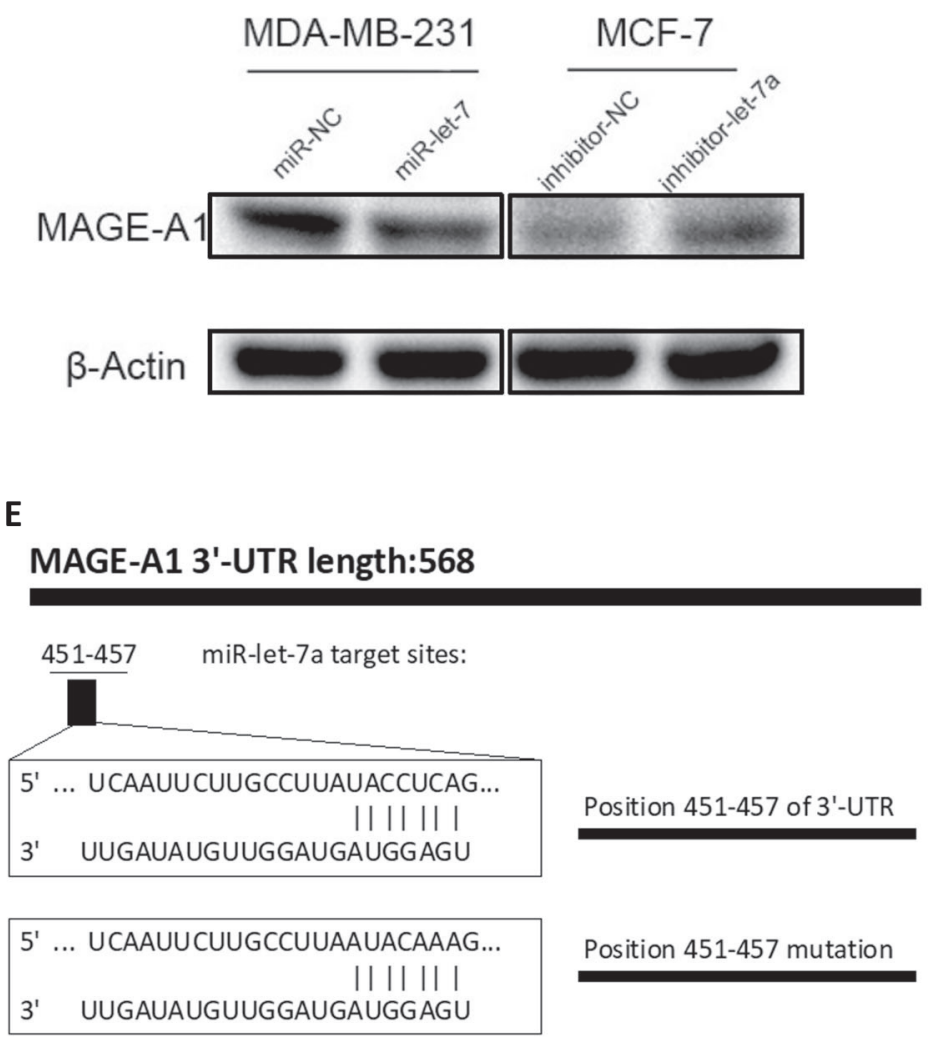

F

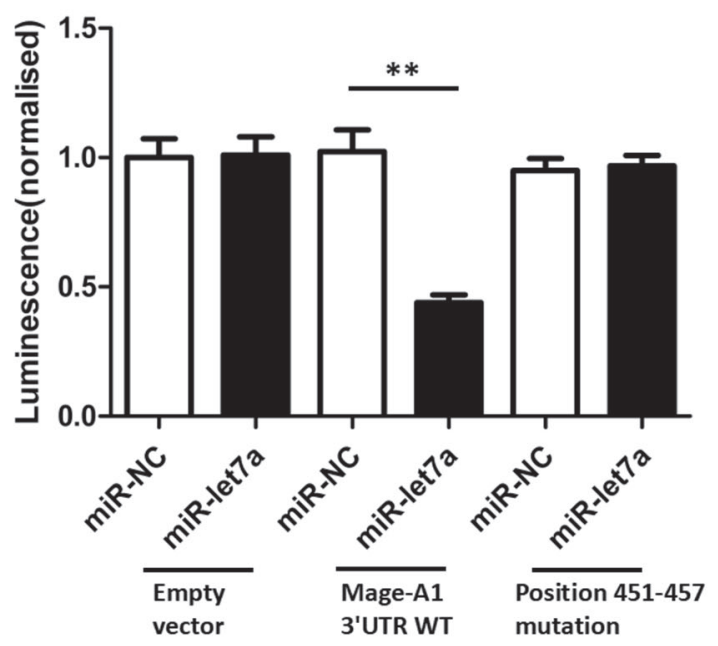

Figure 2. Prediction and validation of MAGE-A1 as the target of let-7a. A) The relative expression level of let-7a in the MCF-7 and MDA-MB-231 cell lines. B, C) The breast cancer cell lines were transiently transfected with let-7a mimics (MDA-MB-231) or let-7a inhibitor (MCF-7). After verifying the transfection efficiency, qRT-PCR was used to investigate the relative expression of MAGE-A1. MAGE-A1 was significantly decreased or increased in response to let-7a mimics or let-7a inhibitor, respectively, compared to their negative controls (mimic NC or inhibitor NC). The graph represents the mean $2-\Delta \Delta C$ t value \pm SD. D) Western blot analysis of the MAGE-A1 protein levels in MDA-MB-231 or MCF-7 cells transfected with let-7a mimic or inhibitor for $48 \mathrm{~h}$ compared to their negative controls. E) Schematic of the putative binding site of let-7a in the MAGE-A1 3'-UTR. A mutant MAGE-A1 3'-UTR construct was tested in parallel. F) Analysis of luciferase activity. Renilla luciferase reporters containing either the wild-type or mutant form of the MAGE-A1 3'-UTR were co-transfected into cos-7 cells with the let-7a mimic or miR-NC. At 48 h post-transfection, Renilla luciferase activity was measured. The results were normalized against firefly luciferase values. (mean $\pm \mathrm{SD} ;{ }^{* *} \mathrm{p}<0.01$ ). 
A

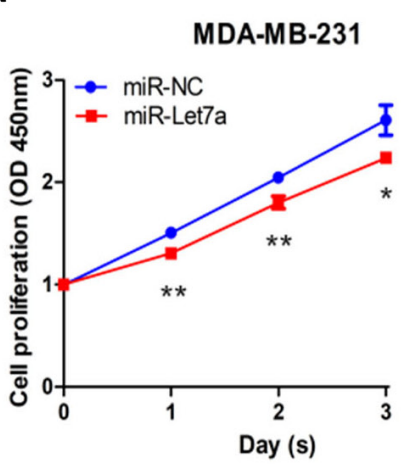

B

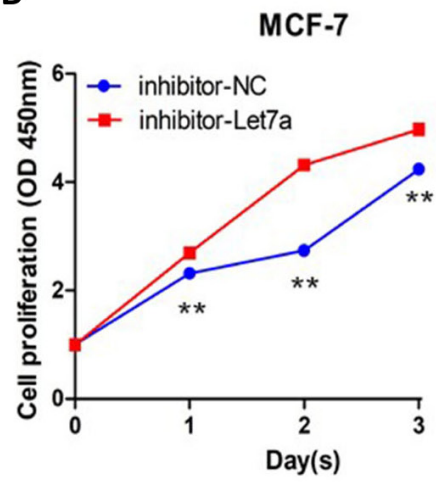

E

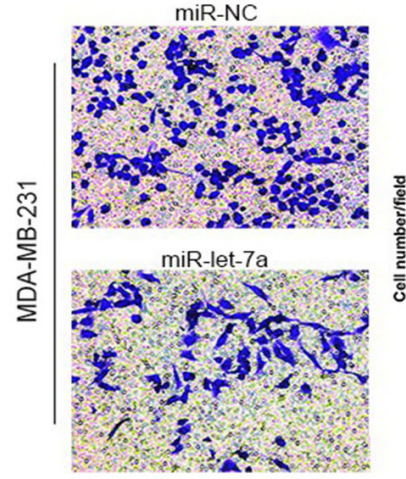

F

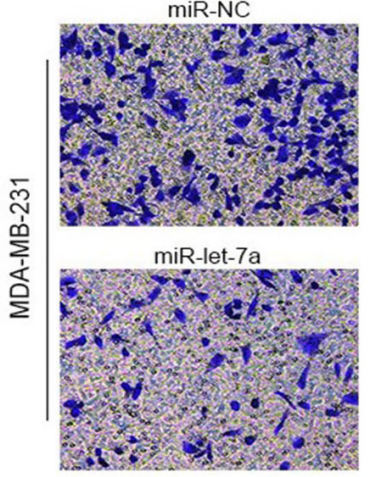

C

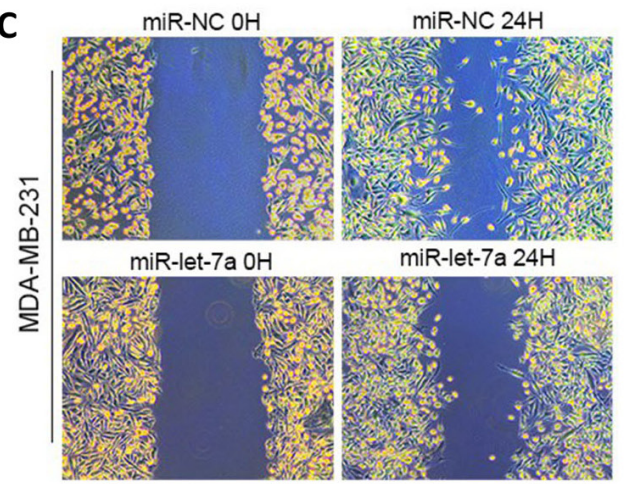

D

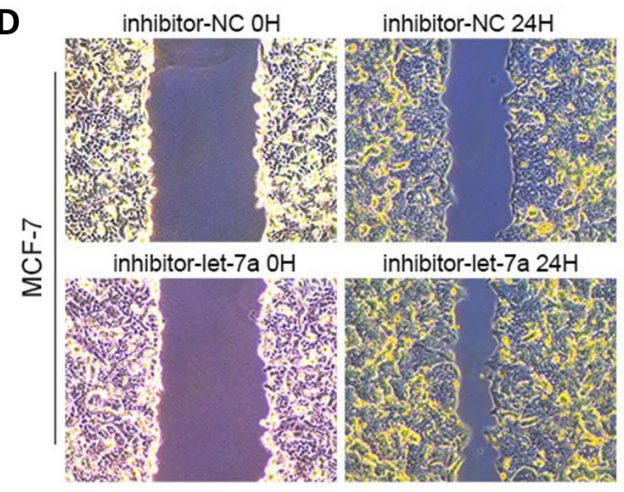

G

H
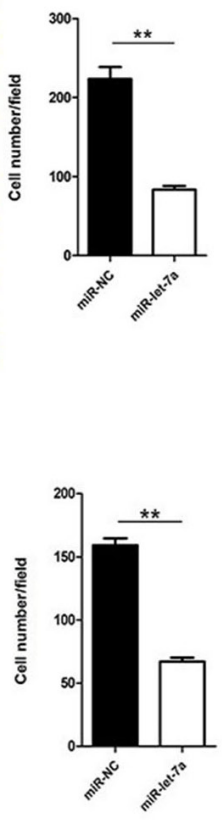
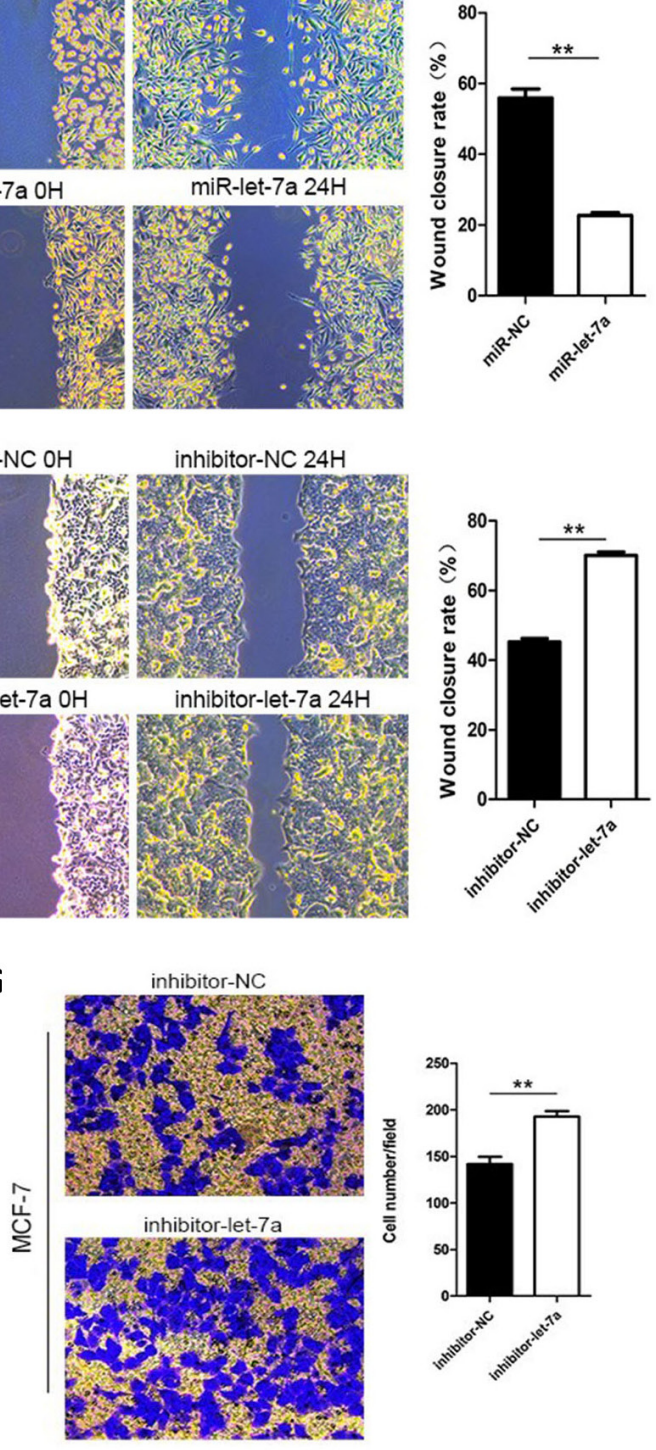

nhibitor-NC
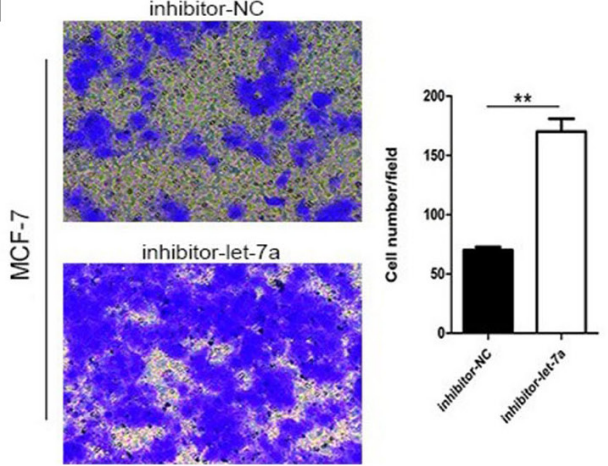

Figure 3. Effects of miR-let7a on tumor cell proliferation, migration and invasion. A, B) CCK-8 assays were performed on days 1, 2 and 3 after transfection of MDA-MB-231 cells with miR-let7a mimic or of MCF-7 cells with let7a inhibitor. C, D) Cell scratch assays were performed at $24 \mathrm{~h}$ after transfection of MDA-MB-231 cells with miR-let7a mimic or after transfection of MCF-7 cells with let7a inhibitor. ImageJ software was used to calculate the mean wound closure rate \pm SD. E, F) Transwell analysis (without matrigel) of MDA-MB-231 cells with miR-let7a mimic or of MCF-7 cells treated with let7a inhibitor. The quantitative analysis is shown in the right panel. G, H) Transwell invasion analysis of MDA-MB-231 cells with miR-let7a mimic or of MCF-7 cells treated with let7a inhibitor (mean $\left.\pm \mathrm{SD} ;{ }^{*} \mathrm{p}<0.05 ;{ }^{* *} \mathrm{p}<0.01\right)$. 
A

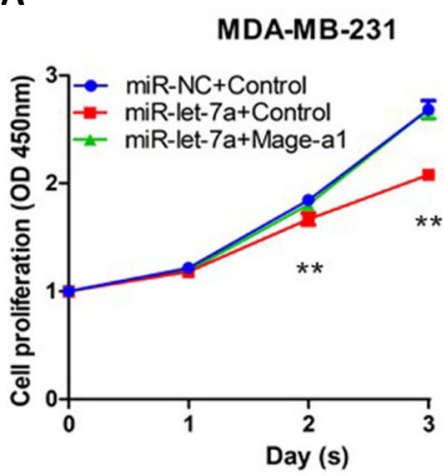

B

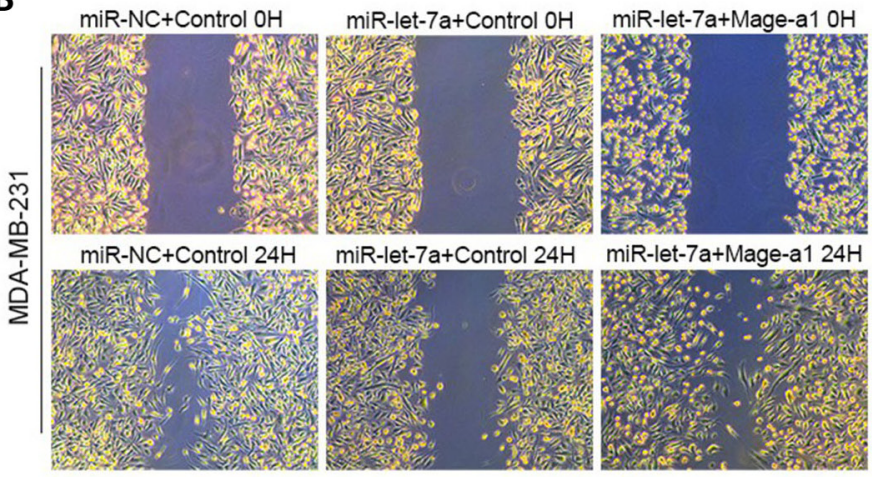

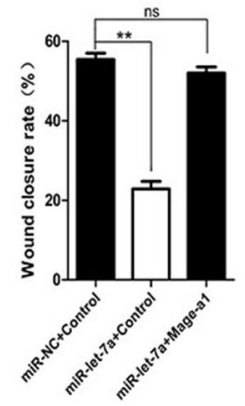

C
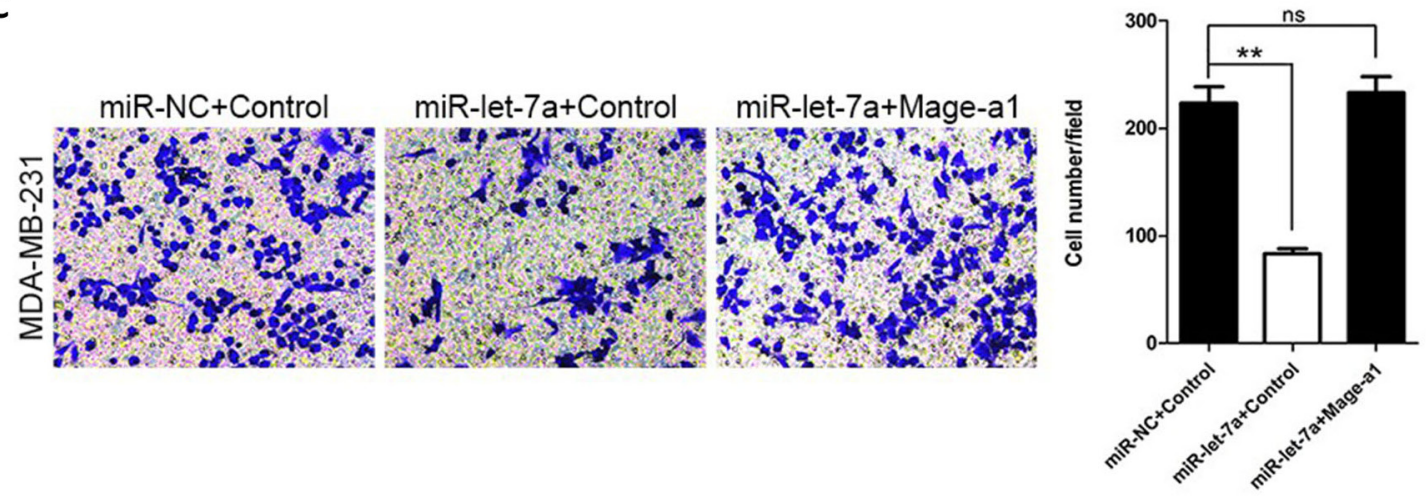

D
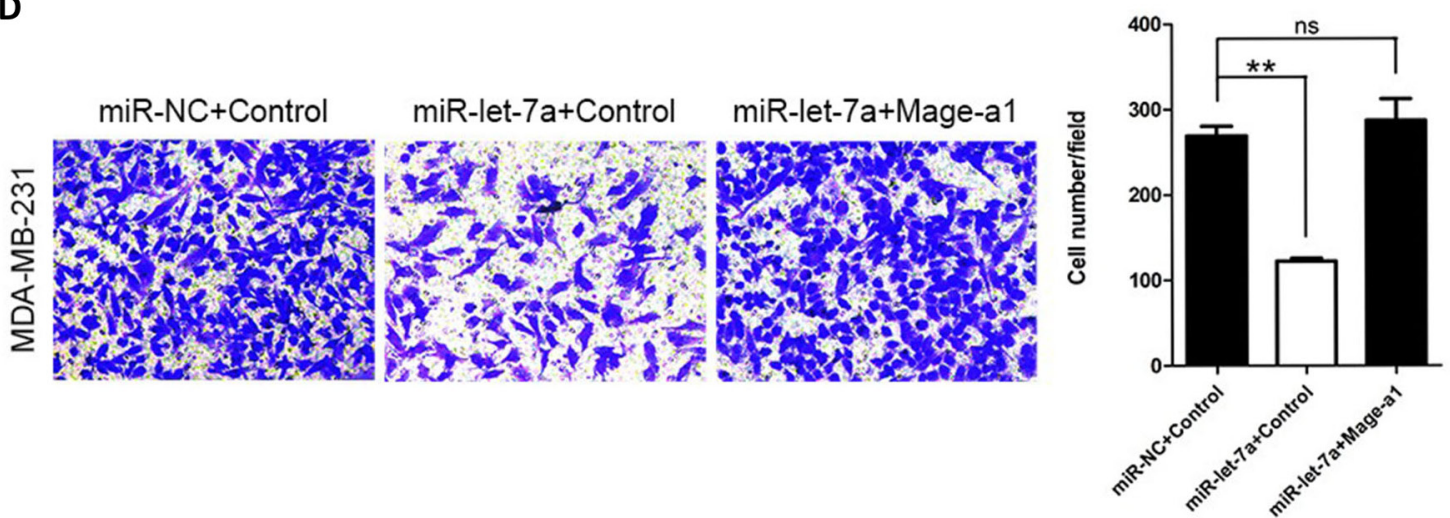

Figure 4. The inhibition effects of let-7a on cell proliferation, migration and invasion were rescued by the co-expression of MAGE-A1 vector in MDAMB-231 cells. A) CCK-8 assays were performed on days 1, 2 and 3 after transfection with the miR-let7a mimic or miR-NC or plus the MAGE-A1 expression vector or control. B) Cell scratch assays were performed at $\mathbf{2 4} \mathbf{h}$ after transfection with the miR-let7a mimic or miR-NC or plus the MAGEA1 expression vector or control. ImageJ software was used to calculate the mean wound closure rate \pm SD. C) Transwell analysis (without matrigel), transfection with miR-let7a mimic or miR-NC or plus the MAGE-A1 expression vector or control. The quantitative analysis is shown in the right panel. D) Transwell invasion analysis, transfection with miR-let7a mimic or miR-NC or plus the MAGE-A1 expression vector or control. The quantitative analysis is shown in the right panel. (mean $\left.\pm \mathrm{SD},{ }^{* *} \mathrm{p}<0.01\right)$.

histological grade III tumors than in patients with histological grade II or I tumors. We also found lower let-7a expression in patients with Her-2-positive tumors and Ki-67 >14\%. Therefore, we conclude that let-7a has an important role in tumor proliferation, migration and invasion.
The expression levels of let-7a and MAGE-A1 in MAGEA1-positive breast cancer tissues significantly negatively correlate. This provides a theoretical basis for the exogenous enhancement of let- $7 \mathrm{a}$ expression to treat breast cancer. To prove the role of let-7a in breast cancer, we then transfected 
let-7a mimics or inhibitor into MDA-MB-231 or MCF-7 cells to induce over-expression or under-expression of let-7a. Exogenous over-expression of let-7a inhibited cell growth as indicated by CCK- 8 assays, whereas low expression levels of let-7a promoted cell growth. Moreover, cell migration and invasion were decreased or increased by the over-expression or under-expression, respectively, of let-7a in MDA-MB-231 or MCF-7 cells, as shown by wound healing and transwell assays.

This is consistent with previous studies and suggests that let- $7 \mathrm{a}$ acts as a tumor suppressor in breast cancer. TargetScan software predicted the binding site of let-7a and MAGE-A1 at bases 451-457 of the 3'UTR region of MAGE-A1 mRNA. Dual-luciferase reporter assays and RT-qPCR and Western blot analyses then established that let-7a specifically binds to this site and inhibits the expression of MAGE-A1 at both the nucleic acid and protein levels. In our final co-transfection experiment, we observed that let-7a inhibited cell proliferation, migration and invasion by targeting MAGE-A1 in a breast cancer cell line.

One miRNA can have multiple mRNA targets, and one mRNA can be targeted by multiple miRNAs [31]. The structures and functions of Let-7 family members and MAGE-A's have many similarities. TargetScan software showed that Let-7 family members have the same predicted binding sites for MAGE-A1 and multiple predicted target binding sites exist between the Let-7 family and MAGE-A's. We therefore deduct that Let-7 family members have important roles in the post-transcriptional regulation of breast cancer by targeting MAGE-A sub-family members.

In breast cancer cells, miRNA gene promoters, including Let-7a-3, tend to be abnormally methylated. However, let-7a-3 methylation is not associated with disease-free survival or overall survival in breast cancer patients [6]. Therefore, we hypothesized that the low expression of Let-7a in breast cancer may be related to the hypermethylation of its gene. However, which signal transduction pathways and signal factors are involved, and the precise relationship between low expression of let-7a in breast cancer and prognosis require further study.

However, our combined results strongly suggest that let-7a acts as a tumor suppressor in breast cancer by targeting MAGE-A1, and the positive let-7a targeting of MAGE-A1 shows great promise as a new strategy in breast cancer treatment.

\section{References}

[1] CHIANG AC, MASSAGUE J. Molecular Basis of Metastasis. N Engl J Med 2008; 359: 2814-2823. https://doi.org/10.1056/ NEJMra0805239

[2] HANAHAN D, WEINBERG RA. Hallmarks of cancer: The next generation. Cell 2011; 144: 646-674. https://doi. org/10.1016/j.cell.2011.02.013
[3] AMBROS V. microRNAs: Tiny regulators with great potential. Cell 2001; 107: 823-826. https://doi.org/10.1016/S00928674(01)00616-X

[4] ESQUELA-KERSCHER A, SLACK FJ. Oncomirs - microRNAs with a role in cancer. Nat Rev Cancer 2006; 6:259-269. https://doi.org/10.1038/nrc1840

[5] MISKA EA. How microRNAs control cell division, differ entiation and death. Curr Opin Genet Dev 2005; 15: 563-568. https://doi.org/10.1016/j.gde.2005.08.005

[6] LIU K, ZHANG C, LI T, DING Y, TU T et al. Let-7a inhibits growth and migration of breast cancer cells by targeting HMGA1. Int J Oncol 2015; 46: 2526-2534. https://doi. org/10.3892/ijo.2015.2949

[7] TAKAMIZAWA J, KONISHI H, YANAGISAWA K, TOMIDA S, OSADA $\mathrm{H}$ et al. Reduced expression of the let-7 microRNAs in human lung cancers in association with shortened postoperative survival. Cancer Res 2004; 64: 3753-3756. https://doi.org/10.1158/0008-5472.CAN-040637

[8] ZHANG HH, WANG XJ, LI GX, YANG E, YANG NM. Detection of let-7a microRNA by real-time PCR in gastric carcinoma. World J Gastroenterol 2007; 13: 2883-2888. https:// doi.org/10.3748/wjg.v13.i20.2883

[9] AKAO Y, NAKAGAWA Y, NAOE T. let-7 microRNA functions as a potential growth suppressor in human colon cancer cells. Biol. Pharm. Bull 2006; 29: 903-906. https://doi. org/10.1248/bpb.29.903

[10] LONG XB, SUN GB, HU S, LIANG GT, WANG N et al. Let7a microRNA functions as a potential tumor suppressor in human laryngeal cancer. Oncol Rep 2009; 22: 1189-1195. https://doi.org/10.3892/or_00000554

[11] JOHNSON SM, GROSSHANS H, SHINGARA J, BYROM $\mathrm{M}$, JARVIS R et al. RAS is regulated by the let-7 microRNA family. Cell 2005; 120: 635-647. https://doi.org/10.1016/j. cell.2005.01.014

[12] SAMPSON VB, RONG NH, HAN J, YANG QY, ARIS V et al. MicroRNA let-7a downregulates MYC and reverts MYCinduced growth in Burkitt lymphoma cells. Cancer Res 2007; 67: 9762-9770. https://doi.org/10.1158/0008-5472

[13] HE XY, CHEN JX, ZHANG Z, LI LC, PENG QL et al. The let-7a microRNA protects from growth of lung carcinoma by suppression of $\mathrm{k}$-Ras and c-Myc in nude mice. J Cancer Res Clin Oncol 2010; 136: 1023-1028. https://doi.org/10.1007/ s00432-009-0747-5

[14] CABALLERO OL, CHEN YT. Cancer/testis (CT) antigens: potential targets for immunotherapy. Cancer science 2009; 100(11): 2014-2021. https://doi.org/10.1111/j.13497006.2009.01303.x

[15] VAN DER BRUGGEN P, TRAVERSARI C, CHOMEZ P, LURQUIN C, DE PLAEN E et al. A gene encoding an antigen recognized by cytolytic T lymphocytes on a human melanoma. Science 1991; 254: 1643-1647. https://doi.org/10.1126/ science. 1840703

[16] BARKER PA, SALEHI A. The MAGE proteins: emerging roles in cell cycle progression, apoptosis, aad neurogenefic disease. J Neurosci Res 2002; 67: 705-712 https://doi. org/10.1002/jnr.10160 
[17] OTTE M, ZAFRAKAS M, RIETHDORF L, PICHLMEIER U, LONING T et al. MAGE-A gene expression pattern in primary breast cancer. Cancer Res. 2001; 61: 6682-6687.

[18] MATKOVIC B, JURETIC A, SPAGNOLI G, SEPAROVIC V, GAMULIN $M$ et al. Expression of MAGE-A and NY-ESO-1 cancer/testis antigens in medullary breast cancer: retrospective immunohistochemical study. Croat Med J 2011; 52: 171177. https://doi.org/10.3325/cmj.2011.52.171

[19] JOOSSE SA, MULLER V, STEINBACH B, PANTEL K, SCHWARZENBACH H. Circulating cell-free cancer-testis MAGE-A RNA, BORIS RNA, let-7b and miR-202 in the blood of patients with breast cancer and benign breast diseases. Br J Cancer 2014; 111: 909-917. https://doi.org/10.1038/ bjc. 2014.360

[20] PASQUINELLI AE, REINHART BJ, SLACK F, MARTINDALE MQ, KURODA MI et al. Conservation of the sequence and temporal expression of let-7 heterochronic regulatory RNA. Nature 2000; 408: 86-89. https://doi. org/10.1038/35040556

[21] ROUSH S, SLACK F. The let-7 family of microRNAs. Trends Cell Biol 2008; 18: 505-516. https://doi.org/10.1016/j. tcb.2008.07.007

[22] ZHOU B, SHAN H, SU Y, XIA K, ZOU R et al. Let-7a inhibits migration, invasion and tumor growth by targeting AKT2 in papillary thyroid carcinoma. Oncotarget 2017; 8: 69746-69755. https://doi.org/10.18632/oncotarget.19261

[23] ERTURK E, CECENER G, TEZCAN G, EGELI U, TUNCA B et al. BRCA mutations cause reduction in miR-200c expression in triple negative breast cancer. Gene 2015; 556, 163-169. https://doi.org/10.1016/j.gene.2014.11.047

[24] WANG L, WANG Y X, ZHANG D Z, FANG XJ, SUN PS et al. Let-7a mimic attenuates CCL18 induced breast cancer cell metastasis through Lin 28 pathway. Biomed Pharmacother 2016; 78: 301-307. https://doi.org/10.1016/j.biopha.2016.01.028
[25] TRANG P, MEDINA PP, WIGGINS JF, RUFFINO L, KELNAR K et al. Regression of murine lung tumors by the let7 microRNA. Oncogene 2010; 29: 1580-1587. https://doi. org/10.1038/onc.2009.445

[26] DOYLE JM, GAO J, WANG J, YANG M, POTTS PR. MAGE-RING protein complexes comprise a family of E3 ubiquitin ligases. Mol Cell 2010; 39: 963-974. https://doi. org/10.1016/j.molcel.2010.08.029

[27] WANG H, SANG M, GENG C, LIU F, GU L et al. MAGE-A is frequently expressed in triple negative breast cancer and associated with epithelial-mesenchymal transition. Neoplasma 2016; 63: 44-56. https://doi.org/10.4149/neo_2016_006

[28] SHANTHA KUMARA HM, GRIECO MJ, CABALLERO OL, SU T, AHMED A et al. MAGE-A3 is highly expressed in a subset of colorectal cancer patients. Cancer Immun 2012; 12: $16-25$.

[29] MENGUS C, SCHUHZ-THATERE, COULOT J, KASTELAN Z, GOLUZA E et al. MAGE-AIO cancer/testis antigen is highly expressed in high-grade non-muscle-invasive bladder carcinomas. Int J Cancer 2013; 132: 2459-2463. https://doi.org/10.1002/ijc.27914

[30] GUO L, SANG M, LIU Q, FAN X, ZHANG X et al. The expression and clinical significance of melanoma-associated antigen-A1,-A3 and-A11 in glioma. Oncol Lett 2013; 6: 5562. https://doi.org/10.3892/ol.2013.1351

[31] HORNSTEIN E, MANSFIELD JH, YEKTA S, HU JK, HARFE BD et al. The microRNA miR-196 acts upstream of Hoxb8 and Shh in limb development. Nature 2005; 438: 671-674. https://doi.org/10.1038/nature04138 